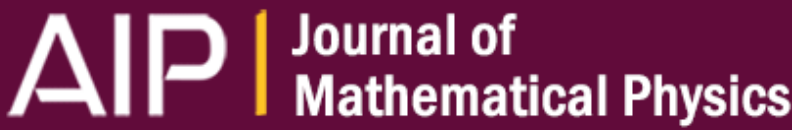

\section{Quantum reverse hypercontractivity}

Toby Cubitt, Michael Kastoryano, Ashley Montanaro, and Kristan Temme

Citation: Journal of Mathematical Physics 56, 102204 (2015); doi: 10.1063/1.4933219

View online: http://dx.doi.org/10.1063/1.4933219

View Table of Contents: http://scitation.aip.org/content/aip/journal/jmp/56/10?ver=pdfcov

Published by the AIP Publishing

\section{Articles you may be interested in}

Time-reversal symmetric resolution of unity without background integrals in open quantum systems

J. Math. Phys. 55, 122106 (2014); 10.1063/1.4904200

Time reversal and $\mathrm{n}$-qubit canonical decompositions

J. Math. Phys. 46, 062104 (2005); 10.1063/1.1900293

Understanding Quantum Computing: Quantum Computation and Quantum Information

Comput. Sci. Eng. 4, 92 (2002); 10.1109/MCISE.2002.1014988

Quantum Computation and Quantum Information

Am. J. Phys. 70, 558 (2002); 10.1119/1.1463744

Quantum grammars

J. Math. Phys. 41, 4508 (2000); 10.1063/1.533357

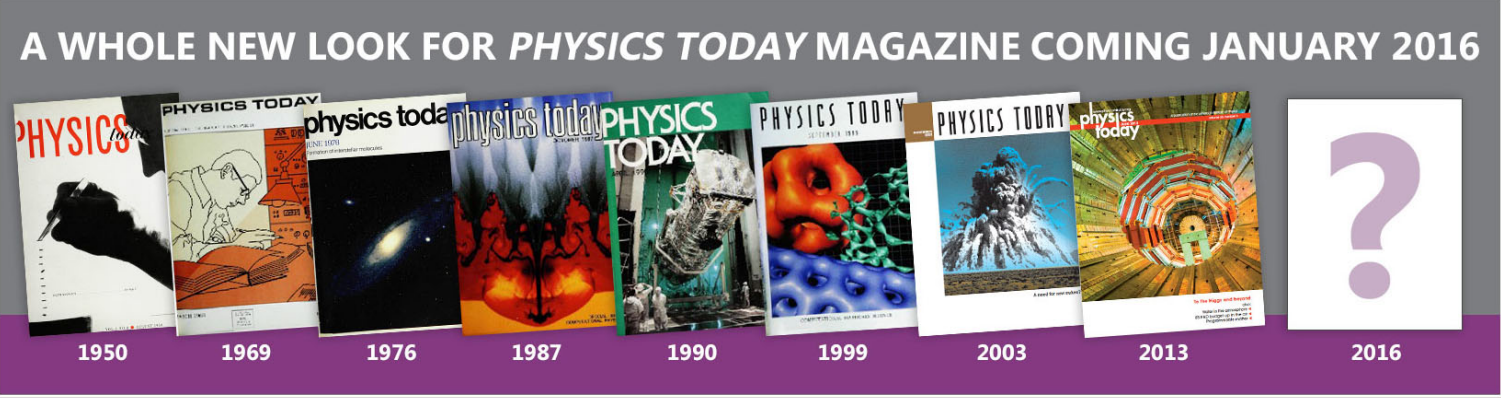




\title{
Quantum reverse hypercontractivity
}

\author{
Toby Cubitt, ${ }^{1}$ Michael Kastoryano, ${ }^{2}$ Ashley Montanaro, ${ }^{3}$ \\ and Kristan Temme ${ }^{4}$ \\ ${ }^{1}$ Department of Computer Science, University College London, London, United Kingdom \\ and Centre for Quantum Information and Foundations, DAMTP, University of Cambridge, \\ Cambridge, United Kingdom \\ ${ }^{2}$ NBIA, Niels Bohr Institute, University of Copenhagen, 2100 Copenhagen, Denmark \\ ${ }^{3}$ School of Mathematics, University of Bristol, Bristol, United Kingdom \\ ${ }^{4}$ Institute for Quantum Information and Matter, California Institute of Technology, \\ Pasadena, California 91125, USA
}

(Received 13 June 2015; accepted 1 October 2015; published online 19 October 2015)

We develop reverse versions of hypercontractive inequalities for quantum channels. By generalizing classical techniques, we prove a reverse hypercontractive inequality for tensor products of qubit depolarizing channels. We apply this to obtain a rapid mixing result for depolarizing noise applied to large subspaces and to prove bounds on a quantum generalization of non-interactive correlation distillation. (C) 2015 AIP Publishing LLC. [http://dx.doi.org/10.1063/1.4933219]

\section{INTRODUCTION}

The theory of hypercontractivity has become an essential tool in disciplines ranging from quantum field theory ${ }^{9}$ to theoretical computer science ${ }^{26}$ and to quantum information theory. ${ }^{15}$ One of the simplest and most well-known, yet also most important, results in this area is hypercontractivity of a certain noise operator acting on the boolean cube $\{0,1\}^{n}$. The noise operator $T_{\gamma}$ is defined by $\left(T_{\gamma} f\right)(x)=\mathbb{E}_{y \sim \epsilon_{\epsilon}}[f(y)]$ for functions $f:\{0,1\}^{n} \rightarrow \mathbb{R}$, where $y$ is distributed such that each bit of $y$ is equal to the corresponding bit of $x$, except with independent probability $\epsilon=(1-\gamma) / 2$. Let $\|f\|_{p}$ be the normalized $\ell_{p}$ norm, $\|f\|_{p}=\left(\frac{1}{2^{n}} \sum_{x \in\{0,1\}^{n}}|f(x)|^{p}\right)^{1 / p}$. Then, it is easy to show that for any $p \geq 1$ and any $\gamma \in[0,1], T_{\gamma}$ is a contraction: $\left\|T_{\gamma}(f)\right\|_{p} \leq\|f\|_{p}$.

However, a stronger result holds.

Theorem 1 (Hypercontractivity for the boolean cube, ${ }^{4,8}$ ). Let $f:\{0,1\}^{n} \rightarrow \mathbb{R}$ and fix $p$ and $q$ such that $1 \leq p \leq q \leq \infty$. Then, for all $\gamma$ such that $0 \leq \gamma \leq \sqrt{(p-1) /(q-1)}$,

$$
\left\|T_{\gamma}(f)\right\|_{q} \leq\|f\|_{p}
$$

Theorem 1 is the key technical result in applications to computer science such as the famous result of Kahn, Kalai, and Linial that every boolean function has an influential variable. ${ }^{11}$ One can generalize Theorem 1 to a quantum (i.e., noncommutative) setting. Here, the natural generalization of the noise operator $T_{\gamma}$ acting on $n$ bits is the qubit depolarizing channel $\mathcal{D}_{\gamma}$ acting on $n$ qubits, applied to each qubit independently. The qubit depolarizing channel is defined by

$$
\mathcal{D}_{\gamma}(f)=(1-\gamma)(\operatorname{tr} f) \frac{I}{2}+\gamma f
$$

where $f \in \mathcal{M}_{2}$ is a linear operator acting on the space of one qubit. The failure of multiplicativity for maximum output $p$-norms of quantum channels implies that it is more challenging to study tensor products of quantum channels than in the classical case. However, the following generalization of Theorem 1 was proven by King, ${ }^{14}$ generalizing a previous result of Montanaro and Osborne.${ }^{16}$ Related special cases were previously shown by Carlen and Lieb ${ }^{6}$ and Biane; ${ }^{3}$ see Ref. 14 for a discussion.

Theorem 2 (Quantum hypercontractivity for the depolarizing channel ${ }^{14}$ ). Let $f \in \mathcal{M}_{2^{n}}$ and fix $p$ and $q$ such that $1 \leq p \leq q \leq \infty$. Then, for all $\gamma$ such that $0 \leq \gamma \leq \sqrt{(p-1) /(q-1)}$,

$$
\left\|\mathcal{D}_{\gamma}^{\otimes n}(f)\right\|_{q} \leq\|f\|_{p} .
$$


In this theorem, $\|\cdot\|_{p}$ is the normalized $\mathbb{L}_{p}$ norm (Schatten $p$-norm), $\|f\|_{p}=\left(\frac{1}{2^{n}} \operatorname{tr}|f|^{p}\right)^{1 / p}$, where $|f|=\sqrt{f^{\dagger} f}$. Theorem 2 has found its own applications, to spectral bounds for local Hamiltonians ${ }^{15}$ and bounds on mixing times for the depolarizing channel. ${ }^{13}$

One can also define the $\ell_{p}$ and $\mathbb{L}_{p}$ "norms" for any $p<1$, though in this case, these functions are no longer actually norms. A result similar in appearance to Theorem 1, but perhaps less well-known, was proven by Borell ${ }^{5}$ for the case where $p$ and $q$ are less than 1 .

Theorem 3 (Reverse hypercontractivity for the boolean cube ${ }^{5}$ ). Let $f:\{0,1\}^{n} \rightarrow \mathbb{R}$ be non-negative and fix $p$ and $q$ such that $-\infty<q \leq p \leq 1$ (if $p<0$, assume that $f$ is strictly positive). Then, for all $\gamma$ such that $0 \leq \gamma \leq \sqrt{(1-p) /(1-q)}$,

$$
\left\|T_{\gamma}(f)\right\|_{q} \geq\|f\|_{p} .
$$

Observe that the inequality in Theorem 3 is reversed as compared with Theorem 1 . Theorem 3 has also found applications in classical computer science, such as isoperimetric inequalities, bounds on correlation distillation, and quantitative versions of Arrow's theorem. . $^{19,17,20}$

Here, we generalize Theorem 3 to the quantum setting, proving the following result.

Theorem 4. Let $f \in \mathcal{M}_{2^{n}}$ be positive semidefinite and let $-\infty<q \leq p \leq 1$ (if $p<0$, assume that $f$ is positive definite). Then, for all $\gamma$ such that $0 \leq \gamma \leq \sqrt{(1-p) /(1-q)}$,

$$
\left\|\mathcal{D}_{\gamma}^{\otimes n}(f)\right\|_{q} \geq\|f\|_{p} .
$$

We apply Theorem 4 to obtain quantum equivalents of some of the main classical applications of reverse hypercontractivity. ${ }^{19,20}$ First, we obtain a "rapid mixing" result for large subspaces. Imagine that $S$ and $T$ are large subspaces of the space of $n$ qubits. One special case of our result is that if we take the uniform mixture on $S$ and apply a certain amount of depolarizing noise to each qubit, the resulting state will have quite a large overlap with $T$. Second, we study a quantum generalization of the problem of non-interactive correlation distillation. ${ }^{18-20}$ Classically, this is a game with $k$ players, where a copy of a string of $n$ random bits is distributed to each player, with independent noise applied to each copy. The goal of the players is to output a single, shared, uniformly random bit. Here, we generalize this by replacing the random bits with a random pure state of $n$ qubits picked from a non-product basis and put limits on the success probability of the players in this more general setting.

\section{PRELIMINARIES}

We let $\mathcal{M}_{d}$ denote the set of complex $d \times d$ matrices. We write $\mathcal{T}_{t}: \mathcal{M}_{d} \rightarrow \mathcal{M}_{d}$ for a general Markovian family of quantum channels generated by a Lindblad operator $\mathcal{L}$,

$$
\mathcal{T}_{t}=e^{-t \mathcal{L}} .
$$

The important special case of the qubit depolarizing channel fits into this picture. We can write $\mathcal{D}_{\gamma}=e^{-t \mathcal{L}}$, where $\gamma=e^{-t}$ and $\mathcal{L}(f)=f-(\operatorname{tr} f) \frac{I}{2}$.

Let $\tau$ be the normalised trace, $\tau(f)=\operatorname{tr} f / d$ for $f \in \mathcal{M}_{d}$. Further, define $\langle f, g\rangle=\tau\left(f^{\dagger} g\right)$. The entropy of $f$ is defined as

$$
\operatorname{Ent}(f)=\tau(f \ln f)-\tau(f) \ln \tau(f) .
$$

For $\mathcal{L}$ the generator of a semigroup, the associated Dirichlet form is

$$
\mathcal{E}_{\mathcal{L}}(f, g)=\tau(f \mathcal{L}(g)) .
$$

For $p \in \mathbb{R}$, the Hölder conjugate $p^{\prime}$ is defined by $1 / p+1 / p^{\prime}=1$.

A quantum channel (completely positive, trace-preserving map) $\mathcal{T}: \mathcal{M}_{d} \rightarrow \mathcal{M}_{d}$ is said to be unital if $\mathcal{T}(I)=I$. $\mathcal{T}$ is said to be primitive if for all density matrices, $\rho$ the $\operatorname{limit}_{\lim } \rightarrow \infty \mathcal{T}^{k}(\rho)$ exists and is equal to some $\sigma>0$ independent of $\rho$. A unital channel $\mathcal{T}$ is said to be reversible if $\tau(f \mathcal{T}(g))=\tau(\mathcal{T}(f) g)$ for all $f, g \in \mathcal{M}_{d}$, i.e., the map is Hermitian with respect to the Hilbert-Schmidt inner product; equivalently, $\mathcal{T}$ 's Kraus operators are Hermitian. (Note that "reversible" is the standard terminology for this property in classical Markov chains, which in our context 
is the special case in which $\mathcal{T}$ is a classical channel (stochastic map). See, e.g., Ref. 23 and references therein for a more extensive discussion of generalisations of Markov chain properties to the quantum setting.)

For unital reversible channels, hypercontractivity of $\mathcal{T}$ (Schrödinger picture) and $\mathcal{T}^{\dagger}$ (Heisenberg picture) is equivalent, although this is not true in general. Since we are only concerned with unital reversible channels in this paper, we work in the Schrödinger picture throughout.

\section{A. Reverse norm inequalities}

We first give reverse versions of various standard $\mathbb{L}_{p}$ norm inequalities. These inequalities seem likely to be well-known, but we include proofs for completeness where these are not easy to find in the literature.

First, we state the reverse Hölder inequality for operators, a proof of which can be found in Ref. 24

Lemma 5. Let $f \geq 0$ be a positive semidefinite operator and let $g>0$ be a positive definite operator. Fix $p$ and $p^{\prime}$ such that $0 \leq p \leq 1$ and $1 / p+1 / p^{\prime}=1$. Then,

$$
\tau(f g) \geq\|f\|_{p}\|g\|_{p^{\prime}}
$$

We will also need the reverse Minkowski inequality.

Lemma 6. Let $f$ and $g$ be positive semidefinite operators and let $p<1$ (if $p<0, f$ and $g$ need to be positive definite). Then,

$$
\|f+g\|_{p} \geq\|f\|_{p}+\|g\|_{p} .
$$

Proof. The proof can be obtained from the reverse Hölder inequality. Let $f, g$ be nonzero positive semidefinite operators. Then,

$$
\begin{aligned}
\|f+g\|_{p}^{p} & =\left\|(f+g)(f+g)^{p-1}\right\|_{1} \\
& =\left\|f(f+g)^{p-1}\right\|_{1}+\left\|g(f+g)^{p-1}\right\|_{1} \\
& \geq\left(\|f\|_{p}+\|g\|_{p}\right) \frac{\|f+g\|_{p}^{p}}{\|f+g\|_{p}},
\end{aligned}
$$

where the inequality follows from the reverse Hölder inequality. The claimed inequality follows by rearranging.

The following lemma gives a variational characterization for $p<1$.

Lemma 7. Let $f$ be positive semidefinite operators and let $p<1$ (if $p<0, f$ needs to be positive definite). Then,

$$
\|f\|_{p}=\inf \left\{\tau(f g): g>0,\|g\|_{p^{\prime}} \geq 1\right\} .
$$

Proof. The proof again follows directly from reverse Hölder.

Finally, we show that the depolarizing channel is expansive for $p<1$.

Lemma 8. Let $f$ be a positive semidefinite operator on $\mathcal{M}_{d}$ and let $p<1$ (if $p<0, f$ needs to be positive definite). Let $\mathcal{T}: \mathcal{M}_{d} \rightarrow \mathcal{M}_{d}$ be a unital quantum channel. Then,

$$
\|\mathcal{T}(f)\|_{p} \geq\|f\|_{p} .
$$

Proof. It is sufficient to show that $\operatorname{tr}|\mathcal{T}(f)|^{p} \geq \operatorname{tr}|f|^{p}$ for $p \geq 0$ and $\operatorname{tr}|\mathcal{T}(f)|^{p} \leq \operatorname{tr}|f|^{p}$ for $p<0$. We write the channel in Kraus form, $\mathcal{T}(f)=\sum_{\alpha} A_{\alpha} f A_{\alpha}^{\dagger}$ for some Kraus operators $\left\{A_{\alpha}\right\}$ and use the following operator Jensen's inequality (see, e.g., Ref. 10): if $\phi$ is a convex function, then

$$
\operatorname{tr} \phi\left(\sum_{\alpha} B_{\alpha}^{\dagger} f B_{\alpha}\right) \leq \operatorname{tr} \sum_{\alpha} B_{\alpha}^{\dagger} \phi(f) B_{\alpha}
$$


for any positive semidefinite operator $f$, and an arbitrary sequence of operators $\left(B_{\alpha}\right)$ such that $\sum_{\alpha} B_{\alpha}^{\dagger} B_{\alpha}=I$. For concave functions, the inequality is reversed. As $\mathcal{T}$ is unital, we have $\sum_{\alpha} A_{\alpha} A_{\alpha}^{\dagger}$ $=I$, so taking $B_{\alpha}=A_{\alpha}^{\dagger}$ and choosing $\phi(x)=|x|^{p}$ (which is concave for $p \geq 0$ and convex for $p \leq 0$ ) yields expansivity of the channel.

\section{PROOF OF THEOREM 4}

Our proof of Theorem 4 will be based on putting together a sequence of small and somewhat more general lemmas; Theorem 4 then becomes a corollary. The overall strategy is to generalize to the quantum setting the proof technique used by Mossel, Oleszkiewicz, and Sen ${ }^{20}$ to prove general reverse hypercontractive inequalities classically. The main technical tool we will need is a quantum generalization of a classical inequality proven by these authors, ${ }^{20}$ which in turn generalizes an inequality of Stroock ${ }^{22}$ and Varopoulos ${ }^{25}$ to $p, q<1$.

Lemma 9 (Quantum Stroock-Varopoulos inequality). Let $\mathcal{T}$ be a unital, reversible, and primitive quantum channel. Let $\mathcal{L}=c_{0}(\mathbf{i d}-\mathcal{T})$, with $c_{0} \in \mathbb{R}_{+}$, denote the generator of a reversible quantum semigroup. Then, for $p \geq q$, with $p, q \in(0,2] \backslash\{1\}$ and positive semidefinite $g \in \mathcal{M}_{d}$, we have

$$
p p^{\prime} \mathcal{E}_{\mathcal{L}}\left(g^{1 / p^{\prime}}, g^{1 / p}\right) \leq q q^{\prime} \mathcal{E}_{\mathcal{L}}\left(g^{1 / q^{\prime}}, g^{1 / q}\right) .
$$

Here, $q^{\prime}$ and $p^{\prime}$ denote the Hölder conjugates of $q$ and $p$, respectively.

Proof. We prove the lemma by showing that the required inequality follows from the classical generalized Stroock-Varopoulos inequality ${ }^{20}$ when considering reversible generators of the form $\mathcal{L}=c_{0}(\mathbf{i d}-\mathcal{T})$. Consider for $a, b \in \mathbb{R}$ the matrix power $g^{a}=\sum_{i} g_{i}^{a}|i\rangle\langle i|$ in the eigenbasis of $g$. Then,

$$
\mathcal{E}_{\mathcal{L}}\left(g^{a}, g^{b}\right)=\tau\left(g^{a} \mathcal{L}\left(g^{b}\right)\right)=\frac{1}{d} \sum_{i, j} g_{i}^{a} g_{j}^{b}\langle i|\mathcal{L}(|j\rangle\langle j|)| i\rangle
$$

Given the Kraus decomposition $\mathcal{T}(f)=\sum_{\alpha} A_{\alpha} f A_{\alpha}^{\dagger}$, we have $\langle i|\mathcal{L}(|j\rangle\langle j|)| i\rangle=c_{0}\left(\delta_{i j}-P_{i j}\right)$, where $P_{i j}=\sum_{\alpha}\left|\left\langle i\left|A_{\alpha}\right| j\right\rangle\right|^{2} \geq 0$ is a doubly stochastic (classical) probability transition matrix. Moreover, since $\mathcal{T}$ is both unital and reversible, we have that $P_{i j}=P_{j i}$. So,

$$
\begin{aligned}
\frac{1}{d} \sum_{i, j} g_{i}^{a} g_{j}^{b}\langle i|\mathcal{L}(|j\rangle\langle j|)| i\rangle & =\frac{c_{0}}{d} \sum_{i, j} g_{i}^{a} g_{j}^{b}\left(\delta_{i j}-P_{i j}\right) \\
& =\frac{c_{0}}{2 d} \sum_{i, j}\left(g_{i}^{a} g_{i}^{b} P_{i j}+g_{j}^{a} g_{j}^{b} P_{i j}-g_{i}^{a} g_{j}^{b} P_{i j}-g_{j}^{a} g_{i}^{b} P_{i j}\right),
\end{aligned}
$$

where the second equality follows from $\sum_{j} P_{i j}=1, P_{i j}=P_{j i}$. We can write this as

$$
\mathcal{E}_{\mathcal{L}}\left(g^{a}, g^{b}\right)=\frac{c_{0}}{2 d} \sum_{i, j} P_{i j}\left(g_{i}^{a}-g_{j}^{a}\right)\left(g_{i}^{b}-g_{j}^{b}\right) .
$$

The claimed inequality then follows from a two-point inequality and then taking an average with respect to the measure $\frac{1}{d} P_{i j}$. It is shown in Ref. 20 (proofs of Lemma 2.4 and Theorem 2.1) that

$$
p p^{\prime}\left(x^{\frac{1}{p}}-y^{\frac{1}{p}}\right)\left(x^{\frac{1}{p^{\prime}}}-y^{\frac{1}{p^{\prime}}}\right) \leq q q^{\prime}\left(x^{\frac{1}{q}}-y^{\frac{1}{q}}\right)\left(x^{\frac{1}{q^{\prime}}}-y^{\frac{1}{q^{\prime}}}\right)
$$

for all $x, y \in \mathbb{R}_{+}$. This implies in particular for the eigenvalues $g_{i} \geq 0$ of $g$ that

$$
\frac{c_{0} p p^{\prime}}{2 d} \sum_{i, j} P_{i j}\left(g_{i}^{\frac{1}{p}}-g_{j}^{\frac{1}{p}}\right)\left(g_{i}^{\frac{1}{p^{\prime}}}-g_{j}^{\frac{1}{p^{\prime}}}\right) \leq \frac{c_{0} q q^{\prime}}{2 d} \sum_{i, j} P_{i j}\left(g_{i}^{\frac{1}{q}}-g_{j}^{\frac{1}{q}}\right)\left(g_{i}^{\frac{1}{q^{\prime}}}-g_{j}^{\frac{1}{q^{\prime}}}\right) .
$$

Reversing the steps now yields the inequality as stated in the lemma.

Remark: The case $q=1$ can be obtained in terms of the appropriate limit. 
By choosing suitable parameters $\left(p=p^{\prime}=2\right)$ in Lemma 9 , we obtain a generalization of a lemma of $\mathrm{Gross}^{8}$ to the quantum setting, for $p<1$. A quantum version of this lemma was previously proven by $\mathrm{King}^{14}$ for $1 \leq p \leq 2$ in the case of unital qubit channels.

Corollary 10 (Quantum Gross's lemma for $p \in(0,2])$. Let $\mathcal{T}$ be a unital, reversible, and primitive quantum channel. Let $\mathcal{L}=c_{0}(\mathbf{i d}-\mathcal{T})$, with $c_{0} \in \mathbb{R}_{+}$, denote the generator of a reversible quantum semigroup. Then, for any $p \in(0,2]$,

$$
\mathcal{E}_{\mathcal{L}}\left(f^{p / 2}, f^{p / 2}\right) \leq \frac{p^{2}}{4(p-1)} \mathcal{E}_{\mathcal{L}}\left(f^{p-1}, f\right) .
$$

This corollary now lets us prove logarithmic Sobolev inequalities for $p<1$, given a logarithmic Sobolev inequality for $p=2$. This condition can be understood as an extension of $\mathbb{L}_{p}$ regularity, but restricted to unital channels. ${ }^{13,21}$ We say that a semigroup generated by $\mathcal{L}$ satisfies a 2 -log-Sobolev inequality with constant $\alpha$ if

$$
\operatorname{Ent}\left(f^{2}\right) \leq \alpha \mathcal{E}_{\mathcal{L}}(f, f) .
$$

Remark: Primitivity of the semigroup is required, as otherwise the log-Sobolev inequality is trivial and the map is not hypercontractive. Indeed, primitivity guarantees that the stationary state of the semigroup is unique and has full rank. For unital semigroups, the maximally mixed state is stationary, hence full rank. To have a non-trivial log-Sobolev inequality, we also have to ensure uniqueness of the fixed point. To see this note that for all reversible channels, one has that $\alpha^{-1} \leq \lambda$, where $\lambda$ is the spectral gap of $\mathcal{L}$, cf. Refs. 21 and 13. For unital channels that are non-primitive, we have that $\lambda=0$ and hence, the constant $\alpha$ diverges.

Lemma 11 (p-log-Sobolev inequalities). Let $\mathcal{T}$ be a unital, reversible, and primitive quantum channel. Let $\mathcal{L}=c_{0}(\mathbf{i d}-\mathcal{T})$, with $c_{0} \in \mathbb{R}_{+}$, denote the generator of a reversible quantum semigroup satisfying a 2-log-Sobolev inequality with constant $\alpha$. For any $p \in(0,2]$,

$$
\operatorname{Ent}\left(f^{p}\right) \leq \frac{\alpha p^{2}}{4(p-1)} \mathcal{E}_{\mathcal{L}}\left(f^{p-1}, f\right)
$$

Proof. Applying the 2-log-Sobolev inequality to $f^{p / 2}$ and then Corollary 10 to $g=f^{p}$, we have

$$
\operatorname{Ent}\left(f^{p}\right) \leq \alpha \mathcal{E}_{\mathcal{L}}\left(f^{p / 2}, f^{p / 2}\right) \leq \frac{\alpha p^{2}}{4(p-1)} \mathcal{E}_{\mathcal{L}}\left(f^{p-1}, f\right)
$$

as required.

The next lemma we will need is a technical claim regarding norm derivatives.

Lemma 12 (Norm derivative). Let $\mathcal{T}_{t}$ be a Markovian family of quantum channels. Let $t: \mathbb{R} \rightarrow$ $\mathbb{R}$ be a differentiable function of $p$ to be defined, and set $f_{t(p)}=\mathcal{T}_{t(p)}(f)$, with $f \geq 0$. Then,

$$
\frac{d}{d p} \ln \left\|\mathcal{T}_{t(p)}(f)\right\|_{p}=\frac{1}{p^{2} \tau\left(f_{t(p)}^{p}\right)}\left(\operatorname{Ent}\left(f_{t(p)}^{p}\right)-p^{2} t^{\prime}(p) \mathcal{E}_{\mathcal{L}}\left(f_{t(p)}^{p-1}, f_{t(p)}\right)\right) .
$$

A variant of this lemma was proven in Ref. 21 . Here, we only consider the case where the reference state in the norm definition is the maximally mixed state. For this special case, the proof can be simplified. ative

Proof. Considering the function $p \mapsto\left\|f_{t(p)}\right\|_{p}=\tau\left(f_{t(p)}^{p}\right)^{1 / p}$, we can directly compute the deriv-

$$
\frac{d}{d p} \ln \left\|f_{t(p)}\right\|_{p}=\frac{1}{p\left\|f_{t(p)}\right\|_{p}^{p}}\left(\frac{d}{d p}\left\|f_{t(p)}\right\|_{p}^{p}-\left\|f_{t(p)}\right\|_{p}^{p} \ln \left\|f_{t(p)}\right\|_{p}\right) .
$$

We now only need to evaluate the derivative $\frac{d}{d p}\left\|f_{t(p)}\right\|_{p}^{p}$. Note that we can write for any diagonalizable operator $f$ and any holomorphic function $h(z)$ that

$$
h(f)=\frac{1}{2 \pi i} \int_{\partial \Delta} h(z) \frac{1}{z-f} d z \text {, where } \Delta \subset \mathbb{C} \text { so that } \operatorname{spec}(f) \subset \Delta,
$$


by the Cauchy integral formula. In particular, for $h(z)=z^{p}$ and $f_{t(p)}$ positive definite, we can choose $\Delta$ to be supported entirely on the right half of the complex plane. When $f_{t(p)}$ is positive semi-definite, we can use a standard continuum argument by first perturbing $f$ so that it is positive definite and then taking the appropriate limit in the end. The derivative can now be evaluated as

$$
\frac{d}{d p} f_{t(p)}^{p}=\frac{1}{2 \pi i} \int_{\partial \Delta}\left(z^{p} \ln z \frac{1}{z-f_{t(p)}}+z^{p} t^{\prime}(p) \frac{d}{d t} \frac{1}{z-f_{t}}\right) d z .
$$

If we choose the branch cut of the complex logarithm to be supported on the negative real axis, the function $z^{p} \ln z$ is holomorphic on $\Delta$. Moreover, we can expand the matrix fraction by the standard formula as

$$
\frac{1}{z-f_{t+\epsilon}}=\frac{1}{z-f_{t}}-\epsilon \frac{1}{z-f_{t}} \mathcal{L}\left(f_{t}\right) \frac{1}{z-f_{t}}+O\left(\epsilon^{2}\right)
$$

up to second order in $\epsilon>0$, from which we see that

$$
\frac{d}{d t} \frac{1}{z-f_{t}}=-\frac{1}{z-f_{t}} \mathcal{L}\left(f_{t}\right) \frac{1}{z-f_{t}} .
$$

We now evaluate the full norm derivative. First, applying Cauchy's integral formula for $z^{p} \ln z$, we then take the normalized trace $\tau$ on both sides of (3). Using the cyclicity of the trace for the second summand, we obtain

$$
\frac{d}{d p}\left\|f_{t(p)}\right\|_{p}^{p}=\tau\left(f_{t(p)}^{p} \ln f_{t(p)}\right)-\frac{t^{\prime}(p)}{2 \pi i} \int_{\partial \Delta} \tau\left(\frac{z^{p}}{\left(z-f_{t(p)}\right)^{2}} \mathcal{L}\left(f_{t(p)}\right)\right) d z .
$$

Note that the second integral can be evaluated by considering the residuum at the second order pole so that

$$
\frac{1}{2 \pi i} \int_{\partial \Delta} \frac{z^{p}}{\left(z-f_{t(p)}\right)^{2}} d z=\operatorname{Res}\left(\frac{z^{p}}{\left(z-f_{t(p)}\right)^{2}}\right)=p f_{t(p)}^{p-1} .
$$

Hence, if we now insert derivative (4) into full expression (2), we obtain

completing the proof.

$$
\begin{aligned}
\frac{d}{d p} \ln \left\|f_{t(p)}\right\|_{p}= & \frac{1}{p^{2}\left\|f_{t(p)}\right\|_{p}^{p}}\left(\tau\left(f_{t(p)}^{p} \ln f_{t(p)}^{p}\right)-\left\|f_{t(p)}\right\|_{p}^{p} \ln \left\|f_{t(p)}\right\|_{p}^{p}\right. \\
& \left.-p^{2} t^{\prime}(p) \tau\left(f_{t(p)}^{p-1} \mathcal{L}\left(f_{t(p)}\right)\right)\right),
\end{aligned}
$$

Combining all these ingredients allows us to make a general statement about when 2-logSobolev inequalities can be lifted to $p$-log-Sobolev inequalities, and thence hypercontractive inequalities, for $0 \leq p \leq 2$.

Theorem 13. Let $\mathcal{T}$ be a unital, reversible, and primitive quantum channel. Let $\mathcal{L}=c_{0}$ (id $-\mathcal{T})$, with $c_{0} \in \mathbb{R}_{+}$, denote the generator of a reversible quantum semigroup $\mathcal{T}_{t}$ satisfying a 2-log-Sobolev inequality with constant $\alpha$.

Let $f \in \mathcal{M}_{d}$ be a positive semidefinite operator and let $-\infty<q \leq p \leq 1$ (if $p<0$, assume that $f$ is positive definite). Then, for all $t$ such that $t \geq \frac{\alpha}{4} \ln ((1-q) /(1-p))$,

$$
\left\|\mathcal{T}_{t}(f)\right\|_{q} \geq\|f\|_{p}
$$

Proof. The proof is essentially the same as the classical proof in Ref. 20. We split into three cases. First, assume that $p>q \geq 0$. Consider a function

$$
t(q)=\frac{\alpha}{4} \ln \frac{1-q}{1-p}
$$

defined on $(0, p]$. Then,

$$
t(p)=0, \quad q^{2} t^{\prime}(q)=\frac{\alpha q^{2}}{4(q-1)} .
$$

Now consider the map $q \mapsto\left\|\mathcal{T}_{t}(f)\right\|_{q}$. By Lemma 12, 


$$
\frac{d}{d q} \ln \left\|\mathcal{T}_{t}(f)\right\|_{q}=\frac{\operatorname{Ent}\left(f_{t(q)}^{q}\right)-\frac{\alpha q^{2}}{4(q-1)} \mathcal{E}_{\mathcal{L}}\left(f_{t(q)}^{q-1}, f_{t(q)}\right)}{q^{2} \tau\left(f_{t(q)}^{q}\right)} \leq 0,
$$

where the inequality follows from Lemma 11. At the right-hand end of the interval, the map evaluates to $\|f\|_{p}$. Therefore,

$$
\left\|\mathcal{T}_{t}(f)\right\|_{q} \geq\|f\|_{p}
$$

or in other words,

$$
\left\|\mathcal{T}_{\frac{\alpha}{4} \ln ((1-q) /(1-p))}(f)\right\|_{q} \geq\|f\|_{p} .
$$

The generalization to $t \geq \frac{\alpha}{4} \ln ((1-q) /(1-p))$ follows from monotonicity of norms by setting $t=\frac{\alpha}{4} \ln ((1-r) /(1-p))$ for some $r \leq q$.

Second, assume that $q<0 \leq p$. Here, we have

$$
\left\|\mathcal{T}_{t}(f)\right\|_{q}=\left\|\mathcal{T}_{t-\frac{\alpha}{4} \ln (1 /(1-p))}\left(\mathcal{T}_{\frac{\alpha}{4}} \ln (1 /(1-p))(f)\right)\right\|_{q} \geq\left\|\mathcal{T}_{\frac{\alpha}{4} \ln (1 /(1-p))}(f)\right\|_{0} \geq\|f\|_{p}
$$

using the first case and that $t \geq \frac{\alpha}{4} \ln ((1-q) /(1-p))$ implies $t-\frac{\alpha}{4} \ln (1 /(1-p)) \geq \frac{\alpha}{4} \ln (1-q)$.

Third, the case $q<p<0$ is proven by a duality argument using Lemma 7 ,

$$
\begin{aligned}
\left\|\mathcal{T}_{t}(f)\right\|_{q} & =\inf \left\{\tau\left(g \mathcal{T}_{t}(f)\right): g>0,\|g\|_{q^{\prime}} \geq 1\right\} \\
& =\inf \left\{\tau\left(f \mathcal{T}_{t}(g)\right): g>0,\|g\|_{q^{\prime}} \geq 1\right\} \\
& \geq \inf \left\{\tau(f h): h>0,\|h\|_{p^{\prime}} \geq 1\right\} \\
& =\|f\|_{p},
\end{aligned}
$$

where we as usual define $p^{\prime}, q^{\prime}$ by $1 / p+1 / p^{\prime}=1 / q+1 / q^{\prime}=1$. The second equality follows from reversibility of $\mathcal{T}_{t}$, while the inequality holds because $t \geq \frac{\alpha}{4} \ln ((1-q) /(1-p))=\frac{\alpha}{4} \ln \left(\left(1-p^{\prime}\right) /(1-\right.$ $\left.q^{\prime}\right)$ ), so for $h=\mathcal{T}_{t}(g),\|h\|_{p^{\prime}} \geq\|g\|_{q^{\prime}}$. This completes the proof.

The special case of the qubit depolarizing channel (Theorem 4) is now simply a corollary.

Theorem 4 (restated). Let $f \in \mathcal{M}_{2^{n}}$ be a positive semidefinite operator and let $-\infty<q \leq p \leq$ 1 (if $p<0$, assume that $f$ is positive definite). Then, for all $\gamma$ such that $0 \leq \gamma \leq \sqrt{(1-p) /(1-q) \text {, }}$

$$
\left\|\mathcal{D}_{\gamma}^{\otimes n}(f)\right\|_{q} \geq\|f\|_{p}
$$

Proof. The assumptions of Lemma 9-11 are all met by $n$ copies, $\mathcal{D}_{\gamma}^{\otimes n}$, of the single qubit depolarizing channel $\mathcal{D}_{\gamma}$. This can be seen by writing $\mathcal{L}_{k}(f)=f-\frac{1}{4} \sum_{\alpha=0}^{3} \sigma_{k}^{\alpha} f \sigma_{k}^{\alpha}$ for the generator of the semigroup acting on the $k^{\prime}$ th qubit, where $\sigma^{\alpha}$ are the Pauli matrices. Then, the overall generator $\mathcal{L}=\sum_{k=1}^{n} \mathcal{L}_{k}$. In addition, it was shown in Ref. 13 that the tensor product of qubit depolarizing channels satisfies a 2-log-Sobolev inequality with constant $\alpha=2$ : $\operatorname{Ent}\left(f^{2}\right) \leq 2 \mathcal{E}_{\mathcal{L}}(f, f)$. We can therefore apply Theorem 13 to the channel $\mathcal{D}_{\gamma}^{\otimes n}$ to obtain the claimed result.

\section{REVERSE HÖLDER AND RAPID MIXING}

We can now collect some corollaries of hypercontractivity for tensor products of qubit depolarizing channels. We first observe a strengthened reverse Hölder inequality.

Corollary 14. Let $f, g \in \mathcal{M}_{2^{n}}$ be positive semidefinite and let $-\infty<q, p \leq 1$ (if $p<0, f$ must be positive definite; if $q<0, g$ must be positive definite). Then, for all $\gamma$ such that $0 \leq \gamma \leq$ $\sqrt{(1-p)(1-q)}$

$$
\tau\left(f \mathcal{D}_{\gamma}^{\otimes n}(g)\right) \geq\|f\|_{p}\|g\|_{q}
$$

Proof. First, observe that it is sufficient to prove the claim for $\gamma=\sqrt{(1-p)(1-q)}$. Otherwise, set $\gamma=\sqrt{(1-p)(1-r)}$ for some $r \geq q$ and observe

$$
\tau\left(f \mathcal{D}_{\gamma}^{\otimes n}(g)\right) \geq\|f\|_{p}\|g\|_{r} \geq\|f\|_{p}\|g\|_{q}
$$

by monotonicity. Assuming $\gamma=\sqrt{(1-p)(1-q)}$, let $p^{\prime}$ satisfy $1 / p+1 / p^{\prime}=1$ and use the reverse Hölder inequality to obtain 


$$
\tau\left(f \mathcal{D}_{\gamma}^{\otimes n}(g)\right) \geq\|f\|_{p}\left\|\mathcal{D}_{\gamma}^{\otimes n}(g)\right\|_{p^{\prime}} .
$$

As $1 /\left(1-p^{\prime}\right)=1-p$, we have $\gamma=\sqrt{(1-p)(1-q)}=\sqrt{(1-q) /\left(1-p^{\prime}\right)}$. By the reverse hypercontractive inequality (Theorem 4 ),

$$
\left\|\mathcal{D}_{\gamma}^{\otimes n}(g)\right\|_{p^{\prime}} \geq\|g\|_{q}
$$

as required to complete the proof.

Following Ref. 19, we now use Corollary 14 to prove the following theorem.

Theorem 15. Let $S$ be a subspace of $\left(\mathbb{C}^{2}\right)^{\otimes n}$ with corresponding projector $\Pi_{S}$, such that $\operatorname{dim} S=\exp \left(-s^{2} / 2\right) 2^{n}$ for some $s \geq 0$. Set $\rho_{S}=\Pi_{S} / \operatorname{dim} S$. Let $M \in \mathcal{M}_{2^{n}}$ satisfy $0 \leq M \leq I, \tau(M)$ $=\exp \left(-t^{2} / 2\right)$ for some $t \geq 0$. Then,

$$
\operatorname{tr}\left(M \mathcal{D}_{\gamma}^{\otimes n}\left(\rho_{S}\right)\right) \geq \exp \left(-\frac{1}{2}\left(\frac{s^{2}+2 \gamma s t+t^{2}}{1-\gamma^{2}}-s^{2}\right)\right) .
$$

Proof. The proof is effectively an immediate consequence of Corollary 14. First, we have

$$
\operatorname{tr}\left(M \mathcal{D}_{\gamma}^{\otimes n}\left(\rho_{S}\right)\right)=\frac{2^{n}}{\operatorname{dim} S} \tau\left(M \mathcal{D}_{\gamma}^{\otimes n}\left(\Pi_{S}\right)\right)=\exp \left(s^{2} / 2\right) \tau\left(M \mathcal{D}_{\gamma}^{\otimes n}\left(\Pi_{S}\right)\right) .
$$

By Corollary 14 , for any $p, q<1$ such that $(1-p)(1-q)=\gamma^{2}$,

$$
\tau\left(M \mathcal{D}_{\gamma}^{\otimes n}\left(\Pi_{S}\right)\right) \geq\left\|\Pi_{S}\right\|_{p}\|M\|_{q} \geq \exp \left(-s^{2} /(2 p)\right) \exp \left(-t^{2} /(2 q)\right),
$$

where we use $0 \leq M \leq I$ in the second inequality. As in Ref. 19 (Theorem 3.4), we maximise the right-hand side by picking

$$
p=\frac{1-\gamma^{2}}{1+\gamma(t / s)}, \quad q=\frac{t}{s} \frac{1-\gamma^{2}}{\gamma+(t / s)}
$$

which yields

$$
\operatorname{tr}\left(M \mathcal{D}_{\gamma}^{\otimes n}\left(\rho_{S}\right)\right) \geq \exp \left(-\frac{1}{2}\left(\frac{s^{2}+2 \gamma s t+t^{2}}{1-\gamma^{2}}-s^{2}\right)\right) .
$$

Note that Theorem 15 holds for any quantum channels satisfying reverse hypercontractivity, not just tensor powers of the depolarizing channel. By fixing parameters in Theorem 15, we obtain the following special case.

Corollary 16. Let $S$ be a subspace of $\left(\mathbb{C}^{2}\right)^{\otimes n}$ with corresponding projector $\Pi_{S}$, such that $\operatorname{dim} S=\sigma 2^{n}$ for some $\sigma$. Let $M \in \mathcal{M}_{2^{n}}$ satisfy $0 \leq M \leq I, \tau(M)=\sigma^{\alpha}$ for some $\alpha \geq 0$. Set $\rho_{S}=$ $\Pi_{S} / \operatorname{dim} S$. Then,

$$
\operatorname{tr}\left(M \mathcal{D}_{\gamma}^{\otimes n}\left(\rho_{S}\right)\right) \geq \sigma^{(\sqrt{\alpha}+\gamma)^{2} /\left(1-\gamma^{2}\right)} .
$$

In the special case $\alpha=1$, this is at least $\sigma^{(1+\gamma) /(1-\gamma)}$.

Let us examine what this corollary is saying. Write $\rho_{\gamma}=\mathcal{D}_{\gamma}^{\otimes n}\left(\rho_{S}\right)$. If $\gamma=0, \rho_{\gamma}$ is maximally mixed, so $\operatorname{tr}\left(M \rho_{\gamma}\right)=\sigma^{\alpha}$. Corollary 16 matches this. More generally, imagine $\sigma, \alpha$, and $\gamma$ are fixed constants. Then, Corollary 16 states that $\operatorname{tr}\left(M \rho_{\gamma}\right)$ is also lower-bounded by a constant, independent of dimension. This is a kind of "rapid mixing" result for tensor products of qubit depolarizing channels: if we start with a random state picked from $S$ and apply depolarizing noise, the resulting state is quite likely to be accepted by the measurement operator $M$, even if, for example, $M$ is the projector onto a subspace orthogonal to $S$.

\section{NON-INTERACTIVE CORRELATION DISTILLATION}

We now apply Theorem 4 to a quantum generalization of the problem of non-interactive correlation distillation. ${ }^{18-20}$ Classically, this problem can be defined in terms of a game involving 
$k$ players. A string of $n$ uniformly random bits is produced and a copy of the $n$-bit string is distributed to each of the players. Each bit in each of the copies is subject to independent noise, being flipped with probability $\epsilon=(1-\gamma) / 2$. Each of the players applies the same boolean function $f:\{0,1\}^{n} \rightarrow\{0,1\}$ to their bit-string. Their aim is that the $k$ output values are all equal to some $y \in\{0,1\}$ such that $y$ is uniformly random.

The following bound on the success probability was shown by Mossel et al. ${ }^{19}$ using reverse hypercontractivity.

Theorem 17 (Mossel et al. ${ }^{19}$ ). For any function $f$, and any noise rate $0 \leq \gamma \leq 1$, the probability that all players output 0 (or 1) is at most

$$
O\left(\left(\frac{e^{c \sqrt{\ln k}}}{k}\right)^{1 / \gamma^{2}-1}\right)
$$

for some universal constant $c$.

The success probability is thus exponentially small in $k$ for any constant noise rate $\gamma$. Theorem 17 is close to optimal: if $f$ is the majority function, for large enough $n$, the probability that all players output the same value is $\Omega\left(k^{1-1 / \gamma^{2}}\right) .{ }^{19}$

We now observe that Theorem 4 allows us to prove a related and more general result in the quantum setting. In the quantum generalization, the players first fix an orthonormal basis $\mathcal{B}$ for $\left(\mathbb{C}^{2}\right)^{\otimes n}$. A state $|\psi\rangle \in\left(\mathbb{C}^{2}\right)^{\otimes n}$ is chosen uniformly at random from $\mathcal{B}$ by a referee. Each of the $k$ players receives a copy of $|\psi\rangle$, with independent depolarizing noise with parameter $\gamma$ applied to each qubit of each copy of $|\psi\rangle$. Each player then applies a "balanced" two-outcome measurement $\{M, I-M\}$ to their state, where $0 \leq M \leq I$ is a positive semidefinite operator such that $\tau(M)=\frac{1}{2}$. It is natural to demand this notion of balance, as even in the noiseless case $(\gamma=1)$, this is necessary in order to obtain equiprobable measurement outcomes, and the players do not necessarily know the noise parameter. As in the classical setting, the goal is for the measurement outcomes to be random and perfectly correlated. That is, either every player should receive the measurement outcome corresponding to $M$ or every player should receive the outcome corresponding to $I-M$, with equal probability of each.

This is a generalization of the classical framework: if the players choose $\mathcal{B}$ to be a product basis for $\left(\mathbb{C}^{2}\right)^{\otimes n}$, the game behaves equivalently to classical non-interactive correlation distillation. In principle, it could be possible for the players to do better by letting $\mathcal{B}$ be a basis of entangled states. However, we show here that an equivalent bound to Theorem 17 can be proven for this more general task.

Theorem 18. For any balanced measurement $\{M, I-M\}$, and any noise rate $0 \leq \gamma \leq 1$, the probability that all players receive outcome $M($ or $I-M)$ is at most

$$
O\left(\left(\frac{e^{c \sqrt{\ln k}}}{k}\right)^{1 / \gamma^{2}-1}\right)
$$

for some universal constant $c$.

Proof. The proof strategy of Ref. 19 (Theorem 3.1) for the classical setting goes through with only minor changes. Assume that the expected probability that all the players output 0 is at least $2 \delta$, i.e.,

$$
\mathbb{E}_{|\psi\rangle}\left[\left(\operatorname{tr} M \mathcal{D}_{\gamma}^{\otimes n}(|\psi\rangle\langle\psi|)\right)^{k}\right] \geq 2 \delta,
$$

where the expectation is over the uniformly random choice of $|\psi\rangle$ from $\mathcal{B}$. Let $S=\operatorname{span}\{|\psi\rangle$ : $\left.\left(\operatorname{tr} M \mathcal{D}_{\gamma}^{\otimes n}(|\psi\rangle\langle\psi|)\right)^{k} \geq \delta\right\}$ and set $\sigma=(\operatorname{dim} S) / 2^{n}$. Using a similar argument to the proof of Markov's inequality, we have $\operatorname{Pr}_{|\psi\rangle}\left[\left(\operatorname{tr} M \mathcal{D}_{\gamma}^{\otimes n}(|\psi\rangle\langle\psi|)\right)^{k} \geq \delta\right] \geq \delta$, and hence, $\sigma \geq \delta$. By the definition of $S$, for any $|\phi\rangle \in S$,

$$
\operatorname{tr}\left[(I-M) \mathcal{D}_{\gamma}^{\otimes n}(|\phi\rangle\langle\phi|)\right] \leq 1-\delta^{1 / k},
$$

so if we write $\rho_{S}=\Pi_{S} / \operatorname{dim} S, \operatorname{tr}\left[(I-M) \mathcal{D}_{\gamma}^{\otimes n}\left(\rho_{S}\right)\right] \leq 1-\delta^{1 / k}$. 
But we can also apply Corollary 16 to $S$ and $I-M$, where $\tau(M)=\frac{1}{2}$ and hence, $\alpha=1$ / $\left(\log _{2} 1 / \sigma\right)$. This implies that

$$
\operatorname{tr}\left[(I-M) \mathcal{D}_{\gamma}^{\otimes n}\left(\rho_{S}\right)\right] \geq \sigma^{\left(1 / \sqrt{\log _{2} 1 / \sigma}+\gamma\right)^{2} /\left(1-\gamma^{2}\right)} .
$$

For any $0 \leq \gamma \leq 1$, the right-hand side is an increasing function of $\delta$, and we therefore have the bound

$$
\operatorname{tr}\left[(I-M) \mathcal{D}_{\gamma}^{\otimes n}\left(\rho_{S}\right)\right] \geq \delta^{\left(1 / \sqrt{\log _{2} 1 / \delta}+\gamma\right)^{2} /\left(1-\gamma^{2}\right)} \geq \delta^{(1 / \sqrt{\ln 1 / \delta}+\gamma)^{2} /\left(1-\gamma^{2}\right)} .
$$

The rest of the proof follows by combining inequalities (5) and (6) to upper-bound $\delta$, exactly as in Ref. 19. Write $v=1 / \gamma^{2}-1$. We will show that if $\delta \geq\left(e^{c \sqrt{\ln k}} / k\right)^{v}$, for a sufficiently large universal constant $c$, then $\delta^{1 / k}+\delta^{(1 / \sqrt{\ln 1 / \delta}+\gamma)^{2} /\left(1-\gamma^{2}\right)}>1$ for large $k$, violating the combination of (5) and (6). First, we have

$$
\delta^{1 / k}>\left(\frac{1}{k}\right)^{v / k}=e^{-(v \ln k) / k}>1-\frac{v \ln k}{k}
$$

We can also expand

$$
\delta^{(1 / \sqrt{\ln 1 / \delta}+\gamma)^{2} /\left(1-\gamma^{2}\right)}=\delta^{\left(\ln ^{-1} 1 / \delta\right) /\left(1-\gamma^{2}\right)} \delta^{2 \gamma\left(\ln ^{-1 / 2} 1 / \delta\right) /\left(1-\gamma^{2}\right)} \delta^{\gamma^{2} /\left(1-\gamma^{2}\right)}
$$

and bound these three parts as

$$
\begin{aligned}
\delta^{\left(\ln ^{-1} 1 / \delta\right) /\left(1-\gamma^{2}\right)} & =e^{-1 /\left(1-\gamma^{2}\right)}, \\
\delta^{2 \gamma\left(\ln ^{-1 / 2} 1 / \delta\right) /\left(1-\gamma^{2}\right)} & =e^{-2 \gamma \sqrt{\ln 1 / \delta} /\left(1-\gamma^{2}\right)} \geq e^{-2 \gamma \sqrt{\nu \ln k} /\left(1-\gamma^{2}\right)}, \\
\delta^{\gamma^{2} /\left(1-\gamma^{2}\right)} & =\delta^{1 / v} \geq \frac{e^{c \sqrt{\ln k}}}{k} .
\end{aligned}
$$

For sufficiently large $c$ (where $c$ depends on $\gamma$, but not on $k$ ), and large enough $k$, the product of these three terms is larger than $(v \ln k) / k$. This implies the desired contradiction completes the proof.

We remark that, classically, reverse hypercontractivity has also been applied to noninteractive distillation of correlations in a more general setting. ${ }^{12}$ Delgosha and Beigi ${ }^{7}$ have shown that "standard" quantum hypercontractivity can be used to put limits on correlation distillation via a quantum generalization of the notion of the hypercontractivity ribbon of Ahlswede and Gács. ${ }^{1}$ It seems likely that the quantum reverse hypercontractive inequality could be applied in a similar way to improve these results, but we do not pursue this further here.

\section{ACKNOWLEDGMENTS}

This work was initiated at the BIRS workshop 15w5098, "Hypercontractivity and Log Sobolev Inequalities in Quantum Information Theory." We would like to thank BIRS and the Banff Centre for their hospitality. We would also like to thank Mark Wilde for pointing out Ref. 24 and an anonymous referee for helpful comments. M.K. was supported by the Carlsbergfond and the Villum foundation. A.M. was supported by the UK EPSRC under Early Career Fellowship EP/L021005/1. K.T. was supported by the Institute for Quantum Information and Matter, a NSF Physics Frontiers Center with support of the Gordon and Betty Moore Foundation (Grants Nos. PHY-0803371 and PHY-1125565). T.S.C. is supported by the Royal Society.

${ }^{1}$ Ahlswede, R. and Gács, P., "Spreading of sets in product spaces and hypercontraction of the Markov operator," Ann. Probab. 4, 925-939 (1976).

2 Beckner, W., "Inequalities in Fourier analysis," Ann. Math. 102, 159-182 (1975).

${ }^{3}$ Biane, P., "Free hypercontractivity," Commun. Math. Phys. 184(2), 457-474 (1997).

${ }^{4}$ Bonami, A., "Étude des coefficients Fourier des fonctiones de Lp(G)," Ann. Inst. Fourier 20, 335-402 (1970).

${ }^{5}$ Borell, C., "Positivity improving operators and hypercontractivity," Math. Z. 180(2), 225-234 (1982).

${ }^{6}$ Carlen, E. and Lieb, E., "Optimal hypercontractivity for Fermi fields and related non-commutative integration inequalities," Commun. Math. Phys. 155, 27-46 (1993).

${ }^{7}$ Delgosha, P. and Beigi, S., "Impossibility of local state transformation via hypercontractivity," Commun. Math. Phys. 332(1), 449-476 (2014); e-print arXiv:1307.2747. 
${ }^{8}$ Gross, L., "Hypercontractivity and logarithmic Sobolev inequalities for the Clifford-Dirichlet form," Duke Math. J. 42, 383-396 (1975).

${ }^{9}$ Gross, L., "Hypercontractivity, logarithmic Sobolev inequalities, and applications: A survey of surveys," in Diffusion, Quantum Theory, and Radically Elementary Mathematics (Princeton University Press, 2006), pp. 45-74.

${ }^{10}$ Hansen, F. and Pedersen, G., “Jensen’s operator inequality,” Bull. London Math. Soc. 35(4), 553-564 (2003).

${ }^{11}$ Kahn, J., Kalai, G., and Linial, N., "The influence of variables on Boolean functions," in Proceedings of 29th Annual Symposium Foundations of Computer Science (IEEE, 1988), pp. 68-80.

${ }^{12}$ Kamath, S. and Anantharam, V., "Non-interactive simulation of joint distributions: The Hirschfeld-Gebelein-Rényi maximal correlation and the hypercontractivity ribbon," in 50th Annual Allerton Conference on Communication, Control, and Computing (Allerton) (IEEE, 2012), pp. 1057-1064.

${ }^{13}$ Kastoryano, M. and Temme, K., "Quantum logarithmic Sobolev inequalities and rapid mixing,” J. Math. Phys. 54, 052202 (2013); e-print arXiv:1207.3261.

${ }^{14}$ King, C., "Hypercontractivity for semigroups of unital qubit channels," Commun. Math. Phys. 328(1), 285-301 (2014); e-print arXiv: 1210.8412.

${ }^{15}$ Montanaro, A., "Some applications of hypercontractive inequalities in quantum information theory," J. Math. Phys. 53, 122206 (2012); e-print arXiv:1208.0161.

${ }^{16}$ Montanaro, A. and Osborne, T., “Quantum Boolean functions,” Chicago J. Theor. Comput. Sci. 16, 1 (2010); e-print arXiv: 0810.2435 .

${ }^{17}$ Mossel, E., “A quantitative Arrow theorem,” Probab. Theory Relat. Fields 154(1-2), 49-88 (2011); e-print arXiv:0903. 2574.

${ }^{18}$ Mossel, E. and O’Donnell, R., "Coin flipping from a cosmic source: On error correction of truly random bits," Random Struct. Algorithms 26(4), 418-436 (2005); e-print arXiv:math.PR/0406504.

${ }^{19}$ Mossel, E., O’Donnell, R., Regev, O., Steif, J., and Sudakov, B., "Non-interactive correlation distillation, inhomogeneous Markov chains, and the reverse Bonami-Beckner inequality," Isr. J. Math. 154(1), 299-336 (2006); e-print arXiv:math/ 0410560.

${ }^{20}$ Mossel, E., Oleszkiewicz, K., and Sen, A., “On reverse hypercontractivity,” Geom. Funct. Anal. 23(3), 1062-1097 (2013); e-print arXiv: 1108.1210

${ }^{21}$ Olkiewicz, R. and Zegarlinski, B., "Hypercontractivity in noncommutative $l_{p}$ spaces," J. Funct. Anal. 161(1), 246-285 (1999).

${ }^{22}$ Stroock, D., An Introduction to the Theory of Large Deviations (Springer-Verlag, 1984).

${ }^{23}$ Temme, K., Kastoryano, M., Ruskai, M., Wolf, M., and Verstraete, F., "The $\chi^{2}$-divergence and mixing times of quantum Markov processes,” J. Math. Phys. 51, 122201 (2010); e-print arXiv:1005.2358.

${ }^{24}$ Tomamichel, M., Berta, M., and Hayashi, M., "Relating different quantum generalizations of the conditional Rényi entropy," J. Math. Phys. 55(8), 082206 (2014); e-print arXiv:1311.3887.

${ }^{25}$ Varopoulos, N., "Hardy-Littlewood theory for semigroups," J. Funct. Anal. 63(2), 240-260 (1985).

${ }^{26}$ de Wolf, R., "A brief introduction to Fourier analysis on the Boolean cube," Theory Comput. Libr. Grad. Surv. 1, 1-20 (2008). 